\title{
Dental Rehabilitation Following Surgical Management of Temporomandibular Joint Ankylosis: An Interdisciplinary Approach
}

\author{
Deepika Patidar ${ }^{1}$, Ramesh R Fry ${ }^{2}$, Suma Sogi ${ }^{3}$, Atul Sharma ${ }^{4}$, Dinesh C Patidar ${ }^{5}$, Aakanksha Sharma $^{6}$
}

\begin{abstract}
Temporomandibular joint ankylosis (TMJ) is a condition affecting mastication, speech, appearance, and oral hygiene to a major extent. The disease is manifested by restriction to complete failure of the TMJ movement due to fibrous or bony union between the condylar head and glenoid fossa. This case report describes a case of a 13-year-old girl with inability to open the mouth along with fractured and discolored anterior teeth due to trauma. Patient was diagnosed as unilateral bony TMJ ankylosis left side and managed by surgical procedure interpositional arthroplasty followed by physiotherapy. Following satisfactory mouth opening, the required dental rehabilitation procedures were done. Combined efforts of pediatric dentists and oral and maxillofacial surgeon with a detailed history, clinical and radiographic assessment helps in correct diagnosis and providing immediate surgical intervention along with the management of associated dental complications in order to reestablish physical and psychological health of the child patient. Keywords: Crown discoloration, Dental trauma, Pulp canal obliteration, TMJ Ankylosis.
\end{abstract}

International Journal of Clinical Pediatric Dentistry (2020): 10.5005/jp-journals-10005-1726

\section{INTRODUCTION}

Temporomandibular joint ankylosis is a disorder that results in restriction of the mouth opening due to fibrous or bony union between the condylar head and glenoid fossa ${ }^{1}$ Trauma is recognized as the most important cause of TMJ ankylosis (57-63\%) in children, while other etiological factors are infections, rheumatoid arthritis, hypoparathyroidism, psoriasis and burns. ${ }^{2}$ It is the most ignored as well as undermanaged problems in children. ${ }^{1}$ Temporomandibular joint ankylosis in children can have a remarkable outcome on physical and psychological well-being due to restricted mouth opening, inability to chew food, compromised aesthetics, poor oral hygiene with resultant dental caries etc., ${ }^{1,2}$

Along with trauma to the TMJ, traumatic injury to the teeth due to fall or any other means could result in crown discoloration and pulp canal obliteration/calcification. The term 'crown discoloration' is broadly applied for any variation, either pink, grey or yellow. ${ }^{3,4}$ Pulp hemorrhage after the dental trauma causes liberation of hemoglobin and erythrocytes which results in hemosiderin (a hemoglobin pigment) penetration in the dental tissues. ${ }^{4}$ About $4-24 \%$ of traumatized teeth develop different level of pulp obliteration which is categorized by the apparent loss of pulpal space in the radiograph. ${ }^{3}$ Pulpal response towards traumatic injuries can be tremendously varied, ranging from immediate pulpal death to slow long-standing pulpal canal obliterations. $^{5}$

Temporomandibular joint ankylosis along with these possible complications following trauma present a unique challenge to pediatric dentists and maxillofacial surgeons in terms of their appropriate management. This case report describes the dental rehabilitation procedures after achieving the adequate mouth opening following surgical management of unilateral TMJ ankylosis in a 13-year-old female.

\section{Case Description}

A 13-year-old female patient came to the Department of Pediatrics and Preventive Dentistry, with the complaint of reduced mouth
${ }^{1,3}$ Department of Pediatrics and Preventive Dentistry, Maharishi Markandeshwar College of Dental Sciences and Research, Mullana, Ambala, Haryana, India

${ }^{2,4-6}$ Department of Oral and Maxillofacial Surgery, Maharishi Markandeshwar College of Dental Sciences and Research, Mullana, Ambala, Haryana, India

Corresponding Author: Deepika Patidar, Department of Pediatrics and Preventive Dentistry, Maharishi Markandeshwar College of Dental Sciences and Research, Mullana, Ambala, Haryana, India, Phone: +91 7988939018, e-mail: drdeepika.prasad@gmail.com

How to cite this article: Patidar D, Fry RR, Sogi S, et al. Dental Rehabilitation Following Surgical Management of Temporomandibular Joint Ankylosis: An Interdisciplinary Approach. Int J Clin Pediatr Dent 2020;13(2):203-205.

Source of support: Nil

Conflict of interest: None

opening and discolored upper front tooth for one year. History revealed that she had a fall from the terrace three years back and did not receive any treatment for the same. There was a gradual reduction in mouth opening for one year, consequently she was not able to eat and maintain oral hygiene properly.

On examination, a reduced mouth opening of $4 \mathrm{~mm}$ was observed with discolored permanent left maxillary central incisor and left mandibular lateral incisor as well (Figs $1 \mathrm{~A}$ and $\mathrm{B}$ ). Due to reduced mouth opening, complete intraoral examination was not possible in the first visit. The patient was referred to Oral and Maxillofacial Surgery Department for the needful treatment. On radiographic investigation with computed tomography (CT) scan, the condyle head was found fused to the articular surface of temporal bone and revealed a bony ankylotic mass with the left TM Joint (Figs 1C and D). Based on these findings patient was diagnosed as unilateral ankylosis of TM Joint left side (Type II Sawhney's Classification).

(-) The Author(s). 2020 Open Access This article is distributed under the terms of the Creative Commons Attribution 4.0 International License (https://creativecommons. org/licenses/by-nc/4.0/), which permits unrestricted use, distribution, and non-commercial reproduction in any medium, provided you give appropriate credit to the original author(s) and the source, provide a link to the Creative Commons license, and indicate if changes were made. The Creative Commons Public Domain Dedication waiver (http://creativecommons.org/publicdomain/zero/1.0/) applies to the data made available in this article, unless otherwise stated. 

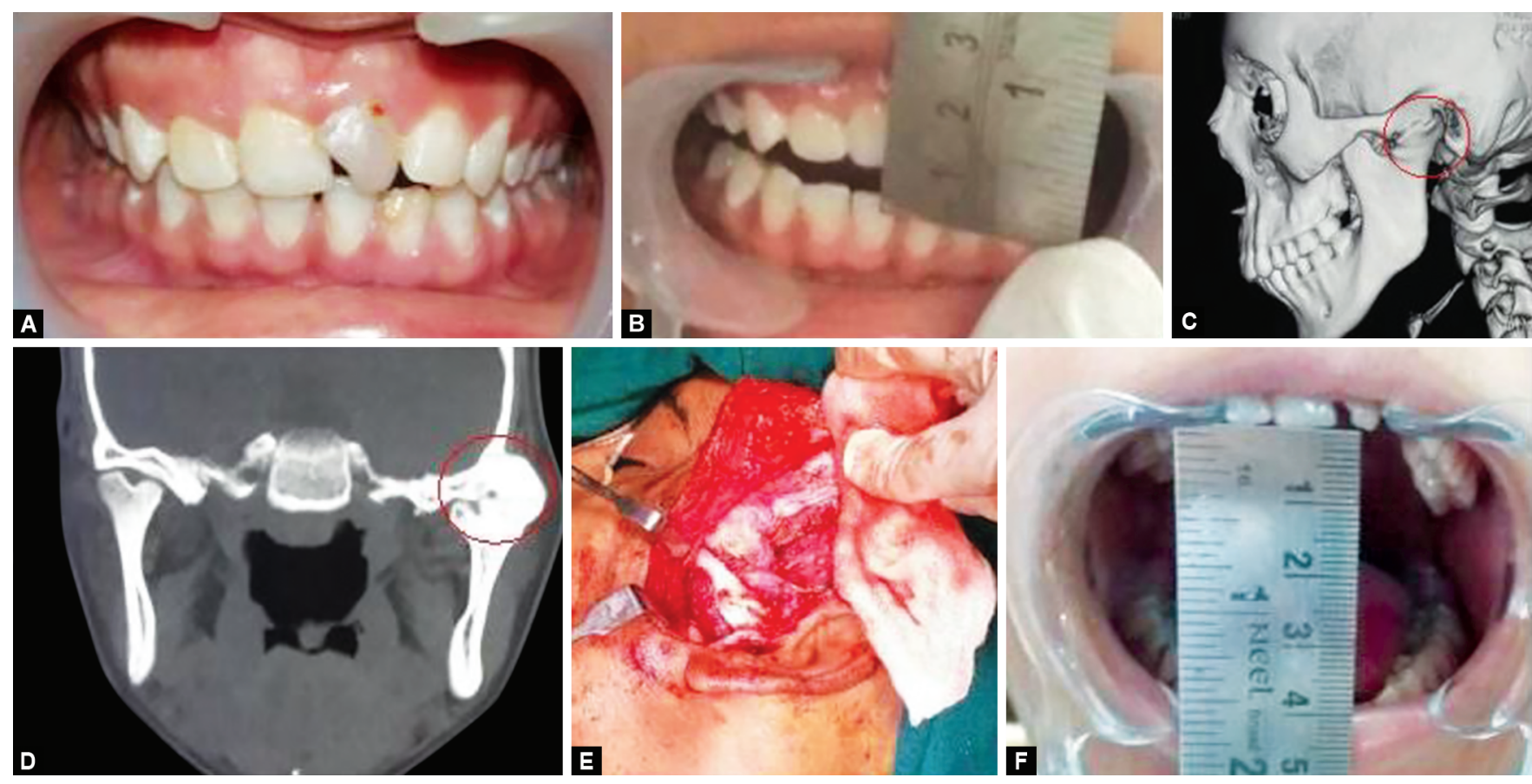

Figs 1A to F: (A) Fractured and discolored 21 and 32; (B) Reduced mouth opening (4 mm); (C) 3D CT scan left side; (D) CT coronal view; (E) Interpositional arthoplasty under G.A; (F) Postoperative mouth opening (40 mm)

After thorough clinical and radiological assessment, a surgical management was planned. Interpositional arthroplasty with temporalis muscle flap on left TMJ was performed under general anesthesia, without the need of coronoidectomy either ipsilateral or contralateral side, as the adequate mouth opening was achieved as per the Kaban's protocol. ${ }^{6}$ Following the surgical procedure, postoperative physiotherapy/mouth opening exercise was done regularly and advised to continue for the period of 6 months. After one month follow-up, mouth opening was satisfactorily maintained to about $40 \mathrm{~mm}$ (Figs $1 \mathrm{E}$ and F). Patient was kept under follow-up for the next six months to one year.

Patient was then referred back to the Dept. of Pediatric and Preventive Dentistry for the further dental treatment procedures. On intraoral examination generalized debris and calculus deposits, fractured and discolored 21 and 32, carious 46 and 36 and deep pits and fissures with respect to (w.r.t.) 172737 were observed (Fig. 1A). Radiovisiography (RVG) investigation revealed periapical radiolucency w.r.t. 21 and 32 while radiolucency involving enamel, dentin and concomitant pulp and periapical region was seen w.r.t. 46. Partial obliteration of the pulp chamber was seen w.r.t 12 while total obliteration of the pulp was noted w.r.t. 11 (Fig. 2A to D). Vitality of maxillary and mandibular anterior was checked by heat test using gutta percha sticks, for which a negative response was given by all the maxillary incisors and mandibular left lateral incisor.

On the basis of these clinical and radiographic findings, complete oral prophylaxis with oral hygiene instructions given to the patient. Pit and fissure sealant was applied on 172737 . Root canal treatment (RCT) was done along with copious normal saline irrigation and intermittent intracanal medicament (metapex) w.r.t. 21 and 32. Simultaneously root canal treatment was also done w.r.t. 46 and 22 and composite resin restoration was done w.r.t. 36. Following RCT, Composite build up was done w.r.t. 21 and 32 and acrylic crown was delivered w.r.t. 21 (Figs $2 \mathrm{E}$ to $\mathrm{G}$ and Fig. 3). Due to pulp calcification and asymptomatic condition 11 and 12 were kept under observation but patient didn't report back for further follow-up visit.

\section{Discussion}

The etiology and treatment of TMJ ankylosis have been well recognized. Trauma is the major causative factor of TMJ ankylosis which results in intra-articular hematoma, consequently results in bone formation leading to the hypomobility. ${ }^{1}$ Difficulty with mastication, compromised aesthetics, speech impairment, poor oral hygiene, dental caries and malocclusion have an intense negative effect on the physical and psychosocial growth of child. ${ }^{2}$

The presented case is of unilateral bony TMJ ankylosis which was due to trauma three years back. Similarly, Hegde et al. ${ }^{7}$ reported history of trauma as a cause of ankylosis in a 12-year-old patient, and Gupta et al. ${ }^{8}$ also found trauma as the most common cause of ankylosis in $90 \%$ of surveyed children. In the present case Interpositional arthroplasty was done under G.A with postoperative physiotherapy to prevent reankylosis, which was consistent with a similar study done by Hegde et al. ${ }^{7}$ and Goel et al. ${ }^{9}$

The present case resulted in satisfactory increase in the mouth opening from $4 \mathrm{~mm}$ to around $40 \mathrm{~mm}$ after the surgery. Following surgical treatment during intraoral examination presence of non vital teeth with discolored and fractured crown w.r.t. 2132 and pulp obliterations w.r.t. 1112 due to trauma were noticed in this patient. It was noted that diffusion of hemosiderin pigment in the dental tissues following trauma can cause change in the crown color. ${ }^{8}$ In a study done by Oginni et al., ${ }^{5}$ out of 168 traumatized teeth, $20.8 \%$ had shown pulpal necrosis, $47.6 \%$ had shown partial while $31.6 \%$ were seen with total pulp obliteration and pulp canal space obliteration was seen more common in concussion and subluxation injuries, although pulpal necrosis was more commonly observed in fractured teeth. The correct mechanism of pulp canal obliteration is not known however is thought to be associated with damage to the neurovascular structure of the pulp during injury. ${ }^{10}$ In the present case 11 and 12 were kept under observation due to pulpal obliteration and asymptomatic condition which was in accordance with McCabe et al. ${ }^{3}$ who concluded in their literature review that 

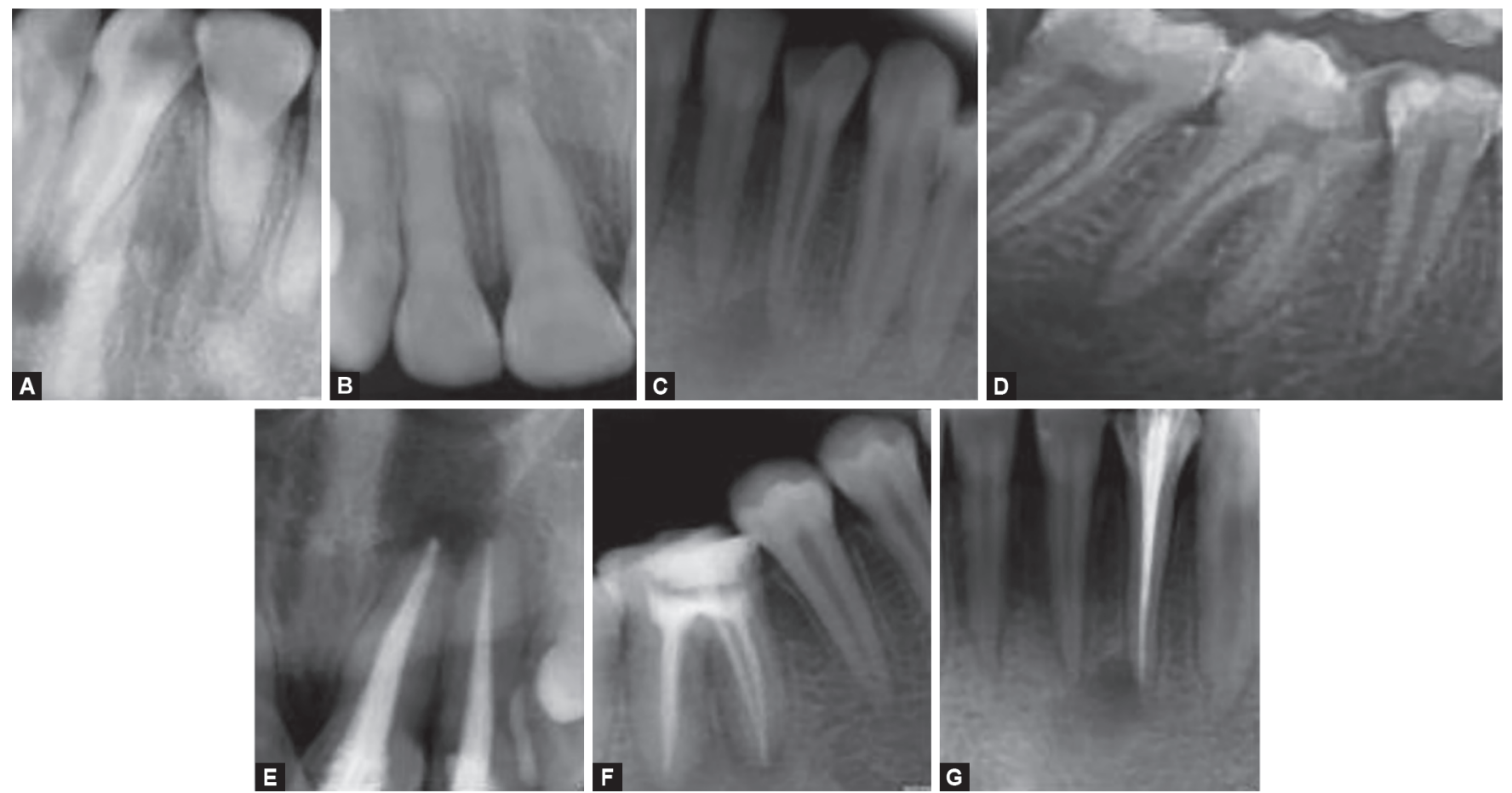

Figs 2A to G: (A) Radiovisiography (RVG) showing periapical radiolucency w.r.t. 21; (B) RVG showing pulp obliteration w.r.t. 11 and 21 ; (C) RVG showing periapical radiolucency w.r.t. 32; (D) Radiograph showing radiolucency involving the pulp and periapical region w.r.t. 46; (E) RVG showing root canal obturation done w.r.t. 21 and 22; (F) RVG showing root canal obturation done w.r.t. 46; (G) RVG showing root canal obturation done w.r.t. 32
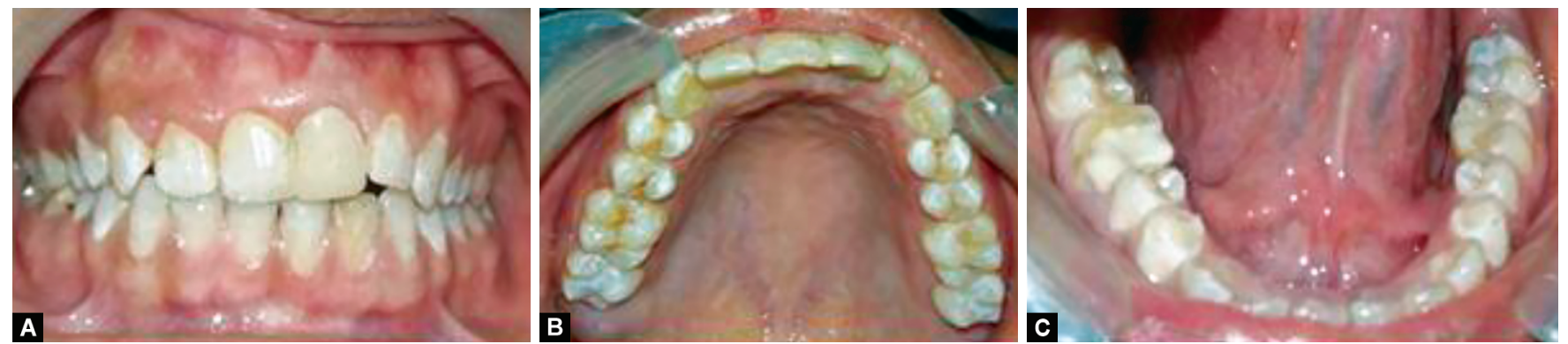

Figs 3 A to C: (A) Postoperative intraoral view showing acrylic crown w.r.t. 21 and composite buildup w.r.t. 22 and 32 followed by root canal treatment; (B) and (C) Pit and fissure sealants given w.r.t. 1727 37, composite build up w.r.t. 36 and post obturation GIC restoration w.r.t. 46

endodontic treatment is indicated when clinical findings and/or definite radiographic signs of periapical pathology is evident. Poor oral hygiene maintenance due to gradual reduction in the mouth opening only to $4 \mathrm{~mm}$ could be the most promising reason for the progression of dental caries in this reported case.

\section{Conclusion/Clinical Significance}

Treatment for TMJ ankylosis in children must be initiated as early as the condition is identified. Combined efforts of pediatric dentist and oral and maxillofacial surgeon in order to provide immediate surgical intervention along with postoperative physiotherapy and appropriate management of all associated dental problems facilitate the positive physical and psychological development of the child.

\section{References}

1. Das UM, Keerthi R, Ashwin DP, et al. Ankylosis of temporomandibular joint in children. JISPPD 2009;27(2):116-120.

2. Shetty $\mathrm{P}$, Thomas A, Sowmya B. Diagnosis of temporomandibular joint (TMJ) ankylosis in children. JISPPD 2014;32(3):266-270. DOI: 10.4103/0970-4388.135848.
3. McCabe PS, Dummer PMH. Pulp canal obliteration: an endodontic diagnosis and treatment challenge. Int Endod J 2012;45:177-197. DOI: 10.1111/j.1365-2591.2011.01963.x.

4. Cardoso M, de Carvalho Rocha MJ. Association of crown discoloration and pulp status in traumatized primary teeth. Dent Traumatol 2010;26(5):413-416. DOI: 10.1111/j.1600-9657.2010.00919.x.

5. Oginni A, Sofowora CA. Pulpal sequelae after trauma to anterior teeth among adult Nigerian dental patients. BMC Oral Health 2007;7(1):11. DOI: 10.1186/1472-6831-7-11.

6. Kaban LB, Bouchard C, Troulis MJ. A protocol for management of temporomandibular joint ankylosis in children. J Oral Maxillofac Surg 2009;67(9):1966-1978. DOI: 10.1016/j.joms.2009.03.071.

7. Hegde RJ, Devrukhkar VN, Khare SS, et al. Temporomandibular joint ankylosis in child: a case report. JISPPD 2015;33(2):166-169. DOI: 10.4103/0970-4388.155136.

8. Gupta VK, Mehrotra D, Malhotra S, et al. An epidemiological study of temporomandibular joint ankylosis. Natl J Maxillofac Surg 2012;3(1):25-30. DOI: 10.4103/0975-5950.102146.

9. Goel M. Management of TMJ ankylosis with interpositional arthoplasty. Clinical Dentistry, Mumbai 2012;6(12):20-24.

10. Faisal A, Gutmann J, Witherspoon DE. Calcific metamorphosis: a challenge in endodontic diagnosis and treatment. Quintessence Interrtational 2001;32(6):447-455. 